\title{
Application and Research on The Model of Distributed Intelligent Cooperative Decision Support System
}

\author{
Peng-Fei Peng, Hao-Tian Cui \\ Electronic Engineering College , Navy University of Engineering , WuHan, China
}

\begin{abstract}
Combined with distributed processing, traditional decision support and collaborative technology, an intelligent distributed collaborative decision support system model is proposed. The architecture, component and functions of the model is described in detail .Analyzed the problem solving process of distributed collaborative decision problem and intelligent collaborative decision-making model is proposed. Finally the intelligent agent model of the system and its application technology is given, which laid the foundation of this new model and its application in military decision making system.
\end{abstract}

\section{Introduction}

DSS (Decision Support System, DSS) is a problem of computer information systems faced by research in the field of advanced stage. With the development of networking, artificial intelligence, distributed processing, system integration, decision support system[1-3] has came into a new stage. Focusing on shortcomings of traditional decision support systems, the model of distributed intelligent collaborative decision support system has been presented. The model fully combined the conclusion of distributed processing[4-6], decision support methods and techniques[7] research, which can meet the multi-level and targeted of decision needed in real life, and multiple users of a variety of application types.

The main body of the model consists of multiple applications, intelligent cooperative decision-making and intelligent agent service nodes. Users can use the Web browser, Windows application client, video the user can be either individual users or workgroups user. System can simultaneously login multiple users of a variety of types, provide a collaborative working environment for the user in order to discuss the result of decisions ,also can make record for results of every decision- making for usage and inquiry in other time.

\section{System Model}

Intelligent distributed collaborative decision supported system is made up of several physically separate massage processing nodes of network collaborative computing environment[8], each node can include application processing, intelligent collaborative decision and intelligent decision agent one or more entities in a proxy service. The model is shown in Figure.1.

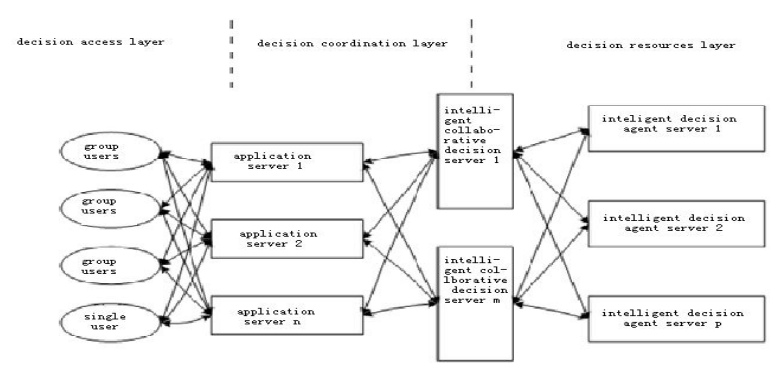

Figure 1 Model of System Architecture

Application server mainly provides users with a variety of human-computer interactive interface and service software, including Web pages, application forms, electronic notebook, E-mail, etc. The diversity of application server determines the users or groups of users will have more choice of ways to participate in decision-making.

Intelligent decision server mainly help to collaborate users on task decomposition of decision making, then look for intelligent decision agents for a problem, and help them to complete all of the decision-making process. Intelligent decision server can manage users to enter, quit, and add, 
delete of server, and send request information of collaborative decision server to users and output the result; receive and process the results of several intelligent agents' decision process; collaborative the work of each application server, responsible for the maintenance of communication functions such as paths, timing, logic of Information transmission.In addition, share real-time global information between intelligent collaborative decision servers of distributed system, collaborate and complete the mission distribution of intelligent decision agents together, and also integrate the results of decisions.

Intelligent decision proxy server accepts the distribute tasks of intelligent collaborative decision server ,activated their own ability of decision dealing, searching for the models, knowledge, data and other content of decision-making, complete the mission of decision-making, and return the results or intermediate results of decision-making to the intelligent decision server.

\section{Intelligent Collaborative Decision Making Model}

In distributed intelligent collaborative decision supported system, there can be more than one intelligent decision server, which distributed in several different nodes. Each intelligent decision server can finish a decision mission on its own, also can complete a decision-making tasks together according to the demand the way to use of users. Each intelligent decision server can connect with multiple application servers, allowed one or more users (teams) joint or independent implement a decision-making process.

As shown in Figure.2, intelligent decision server consists of four basic components: Intelligent user Manager, intelligent problem processor, intelligent decision creator, Communications manager. Intelligent collaborative decision-making independent of network operating systems, database management systems, knowledge database management systems, and the underlying multimedia management systems, adapted to networking distributed systems, workflow :users (groups)log on system through a variety of application servers, inquire the decision or historical decision record of problems with the guide of intelligent user manager. If make decision, systems can distribute the problem according to the specific information of intelligent problem processor, and can make interactive consultation and decomposition of problem and weights case with users(group). After the Atomic issue is reached, users(group), enter the relevant information, solve the atomic issues through each intelligent decision agent of communication manager, result returns to the intelligent collaborative decision server and integrate to build the final result back to the users. In particular, as for group users, sponsor or leader of decision has the final power of decision of input or modify, team members share the information, complete the discussions through the collaboration between intelligent collaborative decision servers or intelligent collaborative decision Server and application server.

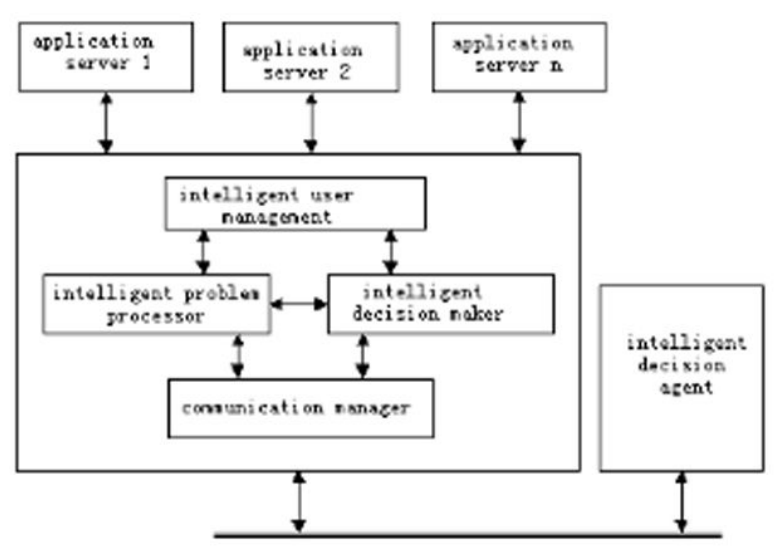

Figure 2 Architecture of Intelligent Cooperative Decision-Making

\subsection{Intelligent user Management}

The intelligent user manager monitor the decision request of users in real-time, responsible for explaining the application sent to intelligence problems processor and forwarding the end result of decision.

Intelligentt user management provide a global view for all users (including which users, which application servers, users are distributed on hich application servers, users' initial information and suggestions to the decision), makes it easy for users to make collaborative decisions. Application servers focusing on specific functions and services of applications provided themselves, and under a unified and collaborated management of Intelligent user manager, its application tools provide all kinds of user interfaces and clients for users to access different applications..

For group of users, sponsor of the decision problem is automatically set as a leader, the leader has the ultimate power with modifying the input information, team member only have the power of discussions and view.

\subsection{Intelligent Problem Solving}

Intelligent problem processor is a core part of intelligent collaborative decision-making service system, its main features are: (1) manage intelligent decision making proxy information, including the position of agent, functions of agent, characteristics of agent and its performance monitoring, call control of agent. (2) Analyzing, processing and managing the problem decomposition and the decomposition of knowledge base.Expert intervention factors of problem decomposition process is also managed by intelligent problem processor. 
Architecture of intelligent problem handling is shown in Figure. 3. Through continuously modify and wealth of agent information, decomposition of file information and knowledge information of intelligent problem processing, which can make the whole system solve different problems. That is the process of intelligent problem solving.

Problem decomposition results are stored in archives, constitute a tree-like hierarchical graph according to the level of decomposition. The leaves of each tree node associated with the intelligent agent, which makes the process of intelligent agents can be processed in parallel. Intelligent problem processor will activate the appropriate intelligent decision agent to collaboratively complete the decisions according to the problem decomposition factor and user's intervention .

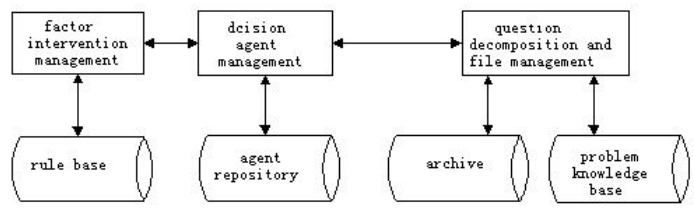

Figure 3 Architecture of Intelligent Agents

\subsection{Intelligent Decision Generate}

The intelligent decision generated part is mainly consists of decision agent result manager and decision generated controller. all decision results finished by intelligent decision agent complete the decomposition of overall mission through collaborative decision server. The result of decision can save as the form of document according to the category of problem decomposition, set the index of file and save in the archive in convenient of inquiry and usage. Besides the result of decision can be sent to the application server in different kinds of forms in order to be showed to users in different forms.

\subsection{Communication Management}

Communication management is mainly complete the communication information management between users and collaborative decision server, collaborative decision server and intelligent decision agent, collaborative decision servers. Communication management is the basic for system to achieve collaborative work, communication is separated to high level and low level. low level use the TCP/IP network communication protocol, the communication mechanism is achieved by agent languages(Java, C\#,etc).The high level communication protocol is consisted of system dedicated orders, which achieved by programming of system developers.

\section{Intelligent Decision Agent Model}

Intelligent decision agent[9] is unified activated using by intelligent decision server, which can solve an atomic problem of decision problem. The features of intelligent decision agent is:1)There is no relation between interpreted mode and system structure. (2) Portability, which means intelligent decision agent can run in every computer system which equipped with interpreter and operating environment, that made intelligent decision agent can be in every nodes of distributed system.(3) Dynamic. System can determine cited decision agent during system running, which can make sure that the revise and update of intelligent decision agent can be reflected in the process of decision-making. By the end of system decision-making, the intelligent decision agent of system will wither away automatically.

The structure of intelligent decision agent is shown as Figure. 4.

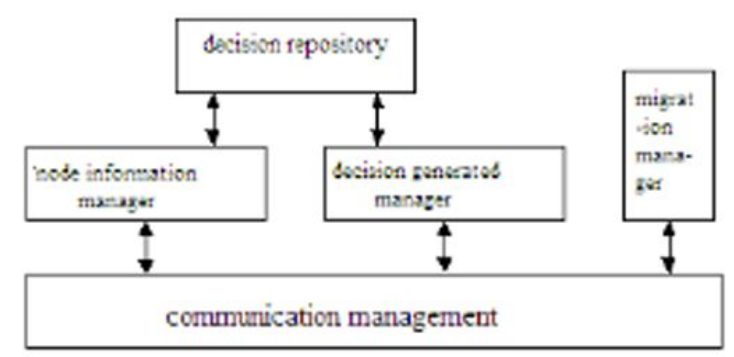

Figure 4 The Structure of Intelligent Decision Agent

The system function of intelligent decision agent is including: (1) Communication management. Its function is similar to communication management in collaborative server, mainly achieve the news and information delivering of intelligent decision agent and collaborative decision service, intelligent decision agent. (2) Migration management. When current decision agent complete its decision-making mission, it will do the decision-making process migration according to the scheduling instructions and related information of intelligent collaborative decision server. it will shut down the agent process, and sends all the information from the current node to the destination node under the instruction of intelligent collaboration server. When all the decision-making agent node complete their missions, the global decision results will be finished by the last intelligent agent server. (3) Decision result generated. All the intelligent decision agent of system will share the public cache provided by intelligent collaborative decision server to save their results, and then the results of decision solving will be achieved by intelligent decision generated agent and intelligent collaborative decision server together. 


\section{Achievement Technology of Model}

The prototype of intelligent distributed collaborative decision support system is the Windows2007 operating system. Using C\# language to developmenta certain type of warship combat simulation and decision support systems in NET environment. Intelligent collaborative decision server is a multi-threaded server established by thread mechanism. The application server of system can provide $\mathrm{B} / \mathrm{S}$ or $\mathrm{C} / \mathrm{S}$ access mode to users. Communication in collaborative processing set Socket based on node protocol by setting Windows service, achieve by using the trigger and recycle receiving of network messages .Information-sharing in collaboration achieved by setting blackboard information buffer in intelligent collaborative serves. Intelligent decision Agent uses Web Service distributed processing technology[10] to implement, by using software or model as the concept of service for intelligent collaborative decision server to dispatch. The treatment of atomic problem used the hybrid reasoning based on cases and rules in intelligent decision agent.

\section{Conclusion}

The distributed intelligent collaborative decision system support model is a new model which is completely different from traditional model of decision support system .Which combined the collaborative, distributed technology with traditional decision support technology, which greatly improved the reliability, portability, reusability, and invulnerability of decision support system. This system model, especially in military decision-making system ,has comprehensive and practical prospect in research and application.

\section{References}

1. Sun Yongyong.Research on Intelligent Decision Support System Based on Multi-Agent[J]. Value Engineering,2014(27):0223-0225.

2. $\mathrm{Yu}$ Weihong.Intelligent Support System for Maritime Search and Rescue Based on JADE[J].Computer Application and Software, 2011,28(8).

3. Yang Fei, Wang Qing, Wu Zhendong.Missile Target Assignment Intelligent Support System Based on GIS[J].Computer Engineering,2010,36(21).

4. Ma Yuxing, Cao Hui, Huang Xiahua.The Application of Multilayer Distributed Processing Technology to Main Engine Remote Control System[J].Ship Electricity Technlogy,2014,34(8).

5. Xue Junjie, Wang Ying, Zhu Jie, Xiao Jiyang, Li Hao.Research Stutus Summary of United States UAV Distributed Processing System[J].Flight Missile,2015.

6. Chen Zhangsheng, Lv Zhiping, Cui Yang, Wang Yupu.Implementation of Distributed Computing with Large-Scale GNSS Data[J].Geomatics and Information Science of Wuhan University,2015,40(3).

7. Li Weigang, Tang Chaosheng, Antonio C. de A. Junior, Liu Peng, Zhang Yaming.A Study on Collborative Decision Making in Air Transportation[J].Complex Systems And Complexity Science,2015,12(2).

8. Liu Yon, Guo Yinzhan, "Expression And Product Design Knowledge Sharing System In Web-Based Collaborative Design,"Jourmal Of Taiyuan University Of Science And Technology,2015,36(4).

9. Wen Bohua, Si Guangya, $\mathrm{Hu}$ Xiaofeng, Luo Pi.Research AndImplementation Of The Third Party Intelligent Agent'S Stretagy Decision-Making Behavior Model[J].Journal Of System Simulation,2005,17(11).

10. Zhu Hongkang, Yu Xueli.Distributed Web Service Mining Technology Based On P2P[J].Computer Engineering,2010,36(9). 